\title{
GESTÃO DOS RECURSOS HÍDRICOS NA SUB-BACIA DO AVANHANDAVA
}

Carlos Roberto Valêncio ${ }^{1}$

Antonio Carlos Carvalho ${ }^{2}$

Toni Jardini ${ }^{3}$

José Roberto Pereira Junior ${ }^{4}$

\section{RESUMO}

Este artigo descreve os trabalhos executados junto à região da sub-bacia crítica do Avanhandava e parte da Bacia Hidrográfica do Turvo Grande. Um conjunto de dados foi coletado in loco, por meio do trabalho de campo e desenvolvido um sistema computacional com recursos georreferenciados de apoio à gestão dos recursos hídricos. Como produto da interação entre o sistema computacional e o trabalho de campo, foi possível traçar a realidade atual de uso dos recursos hídricos e dar subsídios mais precisos para a tomada de decisão com relação às ações preventivas e de recuperação das regiões críticas, além do monitoramento constante e em tempo real. 


\section{INTRODUÇÃO}

A integração de dados georreferenciados com atributos descritivos tornam os SIGs poderosas ferramentas a uma diversidade de aplicações, tais como monitoramento de recursos naturais (SILVA, 2006), monitoramento de incêndios (DEPPE, 2003), planejamento urbano (OLIVEIRA, 2007) entre outras.

Neste trabalho são apresentados os resultados do casamento entre o levantamento in loco de dados de usuários de recursos hídricos e o suporte de um sistema computacional que, por meio de tecnologias emergentes, tem como produto um ambiente de análise amplo, de grande importância para o apoio à gestão ambiental.

O trabalho descrito neste artigo, cujas atividades são divididas entre a confecção do sistema computacional e a coleta de dados in loco, foi executado na bacia do Ribeirão Avanhandava, classificada como crítica, segundo deliberação CBH-TG № 103/2004 de 15/12/2004 (CBH-TG103, 2004). Tal projeto foi aprovado pelo comitê da Bacia Hidrográfica do Turvo Grande, conforme deliberação CBH-TG № 141 de 25/03/2008 (CBH-TG141, 2008).

A coleta dos dados de usuários de recursos hídricos da região abordada foi executada por meio de entrevistas realizadas em cada propriedade. Para tanto, houve a necessidade da confecção de um formulário que reunisse, de maneira abrangente, dados que permitiram caracterizar o perfil dos usuários e, consequentemente, com o suporte da tecnologia SIG desenvolvida, a situação mais precisa dos recursos da região, dado que tem-se constatado que o levantamento dos dados a campo é conflitante com os dados oficiais (FRANCO, 2008)

Por meio dos resultados obtidos, tem sido possível modelar a realidade da subbacia do Avanhandava e abordar, de uma maneira efetiva, a atual situação do local para administrar os recursos hídricos disponíveis e promover ações que melhores se ajustam à recuperação e preservação dos mesmos. 


\section{FUNDAMENTAÇÃO TEÓRICA}

Nessa seção, são apresentados os conceitos básicos referentes a sistema de informação geográfica e banco de dados espaciais, a fim de fornecer embasamento teórico no que diz respeito ao sistema computacional desenvolvido e utilizado para o gerenciamento dos dados coletados a campo.

\subsection{SISTEMA DE INFORMAÇÃO GEOGRÁFICA}

Quando as informações gerenciadas por um sistema computacional necessitam de um contexto espacial para serem corretamente descritas, recuperadas, manipuladas e visualizadas, faz-se necessário o uso de um Sistema de Informação Geográfica (SIG).

No contexto da Ciência da Computação, um SIG é um sistema computacional usado para o entendimento dos fatos e fenômenos que ocorrem no espaço geográfico. A sua capacidade de reunir uma grande quantidade de dados convencionais de expressão espacial, estruturando-os e integrando-os adequadamente, faz com que se tornem ferramentas essenciais para a manipulação das informações geográficas (RISPA, 2000).

As principais vantagens dos SIGs baseados na WEB são: relativo baixo custo de desenvolvimento e implantação, fácil acessibilidade, interface com o usuário mais amigável e intuitiva e, facilidade em integrar diversas fontes de dados disponíveis online (MACLACHLAN, JERRETT et al., 2007) (RAO, FAN, et. al., 2007).

\subsection{BANCO DE DADOS ESPACIAIS}

Um Sistema Gerenciador de Banco de Dados Espaciais (SGBDE) mantém todas as propriedades desejáveis de um gerenciador convencional - atomicidade, consistência, isolamento e durabilidade, para um contexto onde a noção de espaço é necessária. Ele deve incorporar tipos de dados espaciais em seu modelo de dados e de consultas, além 
de suportar eficientemente mecanismos de indexação e junção espaciais (GÜTING, 1994).

Estes requisitos podem ser implementados em um SGBD passível de extensões, como é o caso do SGBD Objeto-Relacional PostgreSQL. A extenção espacial utilizada neste trabalho é a PostGIS, um projeto que usa os recursos de extensão do PostgreSQL para seguir a especificação Simple Feature Specification for SQL, da OGC (Open Geographic Consortium), que define um modelo simples para dados, operações e indexação espacias.

\section{OBJETIVOS}

Um dos objetivos do trabalho foi coletar dados de usuários de recursos hídricos, por meio de entrevistas in loco e, com o suporte oferecido pelo sistema computacional confeccionado, contribuir no suporte à gestão dinâmica dos recursos hídricos e ambientais em sub-bacias, sejam elas preocupantes, em atenção ou críticas, de uso livre aos órgãos públicos de interesse, disponibilizando recursos georreferenciados com tratamento por meio de algoritmos computacionais avançados próprios sobre dados vetoriais e imagens de satélites de média e alta resolução, em ambiente de colaboração online.

Com o advento da Internet, as aplicações SIGs de apoio ao desenvolvimento de políticas públicas e privadas ganharam significativo impulso (NOON, HANKINS, 2001). Diante desse contexto, a confecção do sistema computacional projetado, juntamente com o levantamento dos dados de usuários dos recursos hídricos, coletados in loco pelo trabalho de campo e gerenciados pelo sistema teem como propósito refletir a realidade atual do uso dos recursos hídricos, como a do Ribeirão do Avanhandava, com foco no suporte às ações de recuperação e monitoramento de regiões críticas, abordando os problemas de maneira objetiva. Também o auxiliará no cálculo do balanço hídrico e aprovações de novas outorgas. 


\section{JUSTIFICATIVA}

Os riscos das degradações dos mananciais e nascentes decorrem das características da urbanização dos municípios da bacia, e das finalidades do uso dos recursos hídricos nos diversos segmentos do processo de industrialização, comércio e agropecuária. Todas essas atividades que se processam em moldes preponderantemente inadequados e sem controle da maneira com que os recursos hídricos estão sendo utilizados e explorados, desconhecendo-se o volume utilizado e não os confrontando com a realidade da capacidade máxima permitida. Nota-se também que rios e nascentes estão sendo dizimados, perdendo espaço para atividades agrícolas e outros fatores de prejuízos constantes junto a estes recursos.

Na Figura 1 é ilustrada uma das possíveis situações ocorridas com freqüência, onde houve a tentativa de recuperação da mata ciliar de um rio, mas de modo inadequado, não proporcionando a recuperação da respectiva mata. Outro exemplo é ilustrado na Figura 2 onde se efetiva a tentativa de recuperação de uma nascente, mas em vão, pois a ausência de suporte técnico faz com que o proprietário recorra à ação do vento para retirar a água (moinho a vento) de um poço com objetivo de dar de beber ao gado.

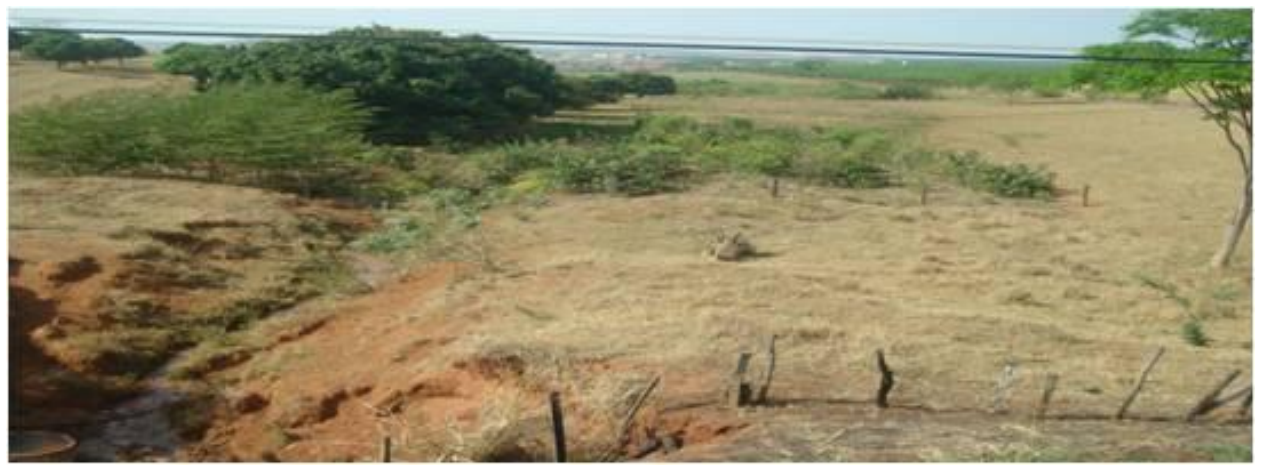

Figura 1 - Rio desprovido de mata ciliar, mesmo após tentativa de recuperação 

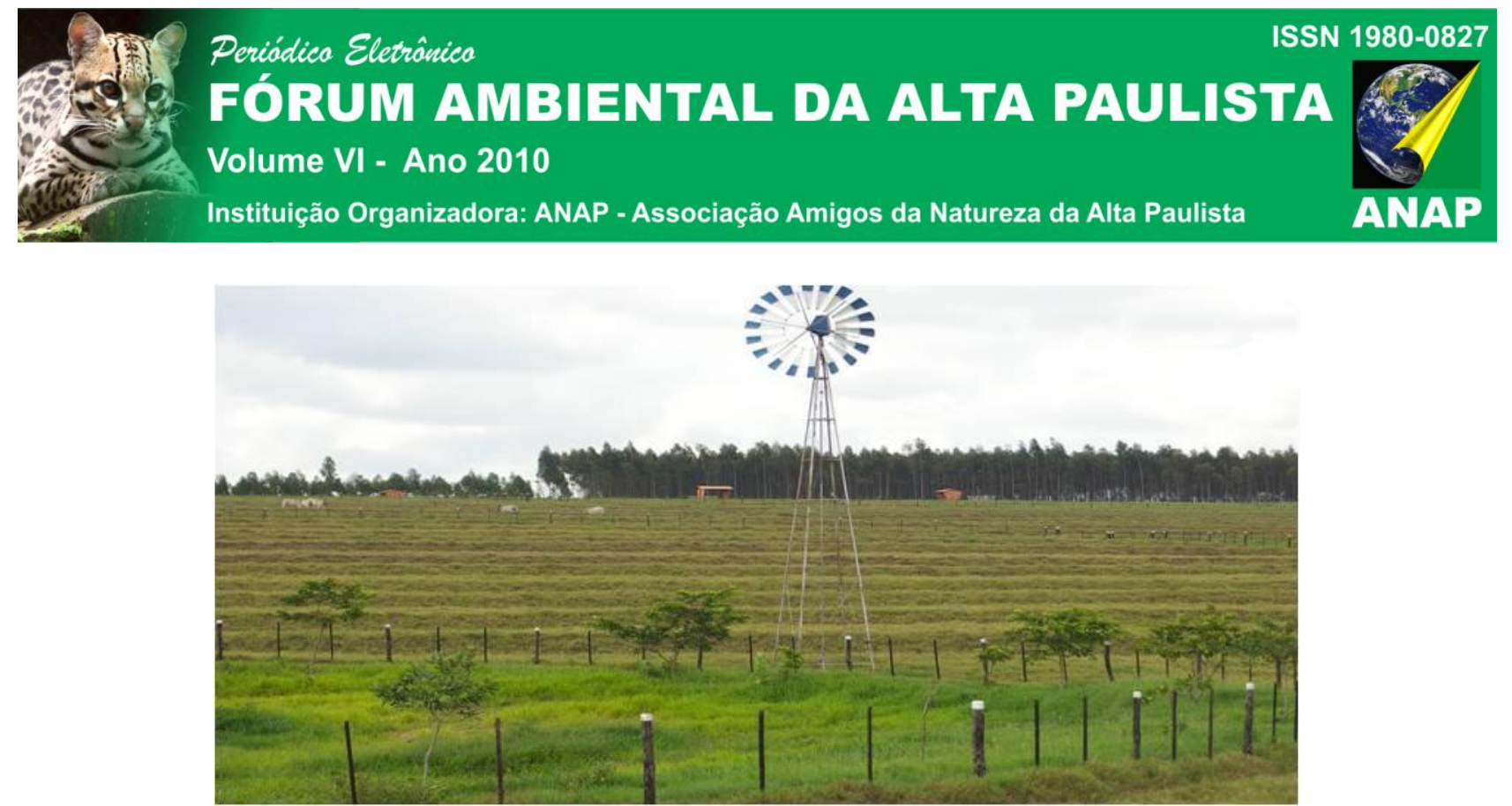

Figura 2 - Tentativa de recuperação de nascente sem sucesso com conseqüente obtenção de água por moinho de vento

Pouco se sabe sobre o destino destes recursos nos diversos segmentos do mercado. A prioridade é fornecer água para consumo humano que abastece os núcleos urbanos.

Devido à necessidade de saber o montante gasto em todos os segmentos do uso de recursos hídricos, faz-se necessário um monitoramento completo a fim de gerenciar esse consumo com a pretensão de evitar um colapso de abastecimento.

São necessárias ações capazes de promover a recuperação ambiental, assegurar a preservação e iniciar a recuperação ambiental dos recursos hídricos da bacia, dentro de uma perspectiva de desenvolvimento sustentável.

A partir do conhecimento das variáveis que incidem sobre esse problema que envolve a área, é possível propor ações orientando diretrizes de proteger e assegurar um futuro sem comprometimento para a população que reside na Sub-Bacia do Avanhandava. Uma vez conhecida a realidade da sub-bacia do Avanhandava, vem sendo possível sugerir um modelo diferenciado para administrar os recursos hídricos disponíveis e programas de uso e racionalização dos mesmos com a pretensão de minimizar o problema.

\section{METODOLOGIA}


O desenvolvimento do projeto de construção do sistema computacional e realização do trabalho de campo para coleta de dados seguiu um roteiro, organizado em seis etapas.

Para o trabalho de campo, em primeiro instante, foi divulgado junto às entidades afins, o motivo da execução do trabalho.

Posteriormente, foram definidos os parceiros para a realização do projeto, por meio de contatos com autoridades dos municípios, sob a supervisão do contratante para divulgar o propósito do projeto, visando obter apoio dos mesmos.

Para o levantamento dos dados, a equipe de trabalho de campo visitou todas as propriedades da região, totalizando 181 cadastros de recursos hídricos, incluindo poços semi-artesianos, captação de superfície, poço simples (caipira) e minas. Além do levantamento de dados de usuários de recursos hídricos, tipo de cultura cultivada, irrigação e outros, as unidades de recursos hídricos foram georreferenciadas.

$\mathrm{Na}$ etapa de desenvolvimento do sistema computacional, responsável por armazenar e exibir os dados dos questionários a respeito das informações sobre usuários de recursos hídricos coletados no trabalho de campo e dar suporte às ações de preservação e recuperação das regiões críticas, com toda funcionalidade de geração de relatórios dinâmicos em ambiente georreferenciado, foi utilizado o processo de desenvolvimento numa perspectiva de Processo Unificado - PU (LARMAN).

Depois de implantado, a coleta in loco de dados realizada pelo trabalho de campo, juntamente com o suporte oferecido pelo sistema computacional desenvolvido, possibilitou a criação de modelos para gestão de outorga dos recursos hídricos da região abordada e traçaram um perfil amplo dos usuários. Tais informações estão sendo inseridas em programa abrangente de racionalização de uso dos recursos hídricos, de acordo com metas estabelecidas pelo órgão que deliberou a criticidade da sub-bacia.

\section{RESULTADOS}


A coleta dos dados e o suporte oferecido pelo sistema computacional permitiram, dentre inúmeros produtos dessa interação:

- Avaliar o consumo de água em suas finalidades e uso, através de relatórios dinâmicos gerados em um ambiente georreferenciado;

- Analisar e monitorar as demandas cadastradas em função da disponibilidade hídrica e monitoração estimada do recurso hídrico, como visto na figura 3;

- Diagnosticar dados individualizados por curso de água, sub-bacia, município, polígono arbitrário (porção de região selecionada) e tipo de uso, de forma a possibilitar um balanço hídrico de cada variável proposta, visto na figura 4;

- Disponibilizar os cadastros dos usuários que já estão implantados no município, identificando e caracterizando os usos existentes (local de captação, manancial, vazão retirada, tipo de equipamento, coordenadas UTM, utilidade, razão social, etc.);

- Disponibilizar a estimativa de demanda em tempo real da sub-bacia, fazendo comparações com a população existente;

- Apresentar a disposição espacial das informações em interface georreferenciável associada aos dados obtidas do sistema online de cadastro dos recursos hídricos;

- Oferecer infraestrutura necessária para análises dos dados baseadas nas posições espaciais dos recursos em questão, gerando relatórios dinâmicos com auxílio da interface georreferenciável.

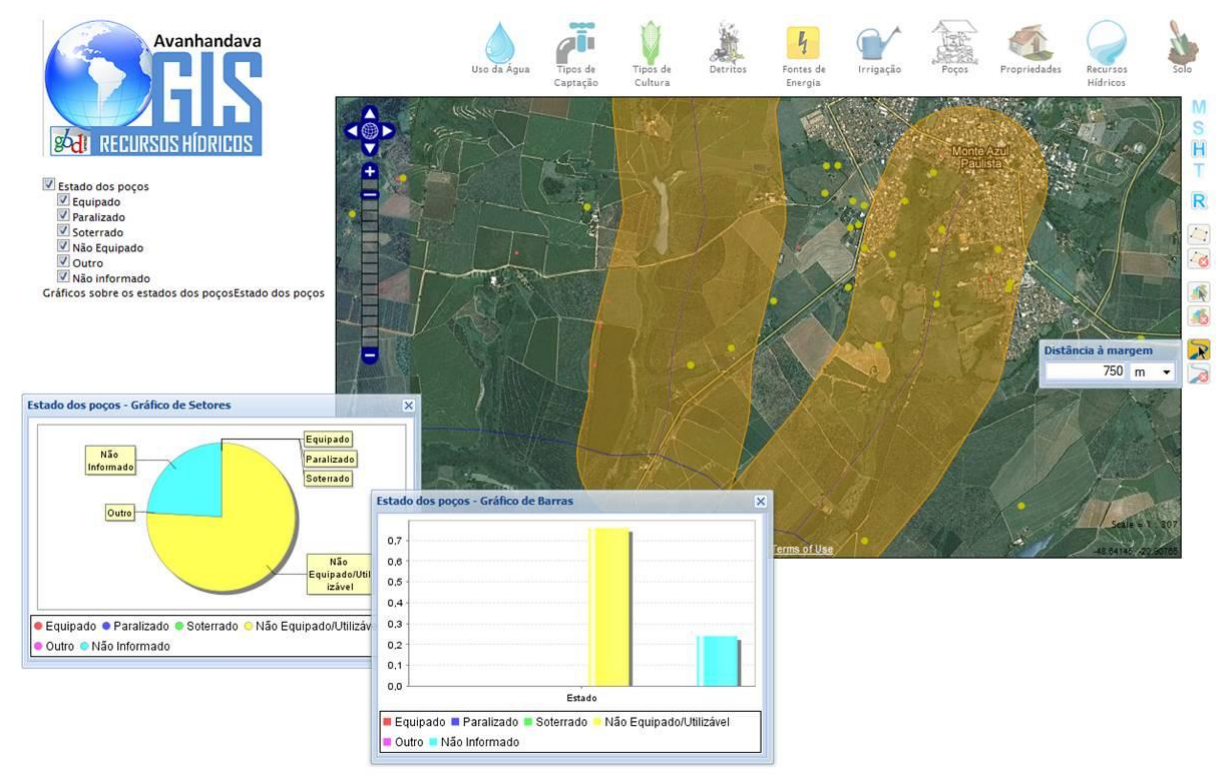




\section{Periódica Eletrânica

Figura 3. Exemplo de relatório de estado de poços, extraído de dados referentes ao raio de $750 \mathrm{~m}$ de distância do rio selecionado

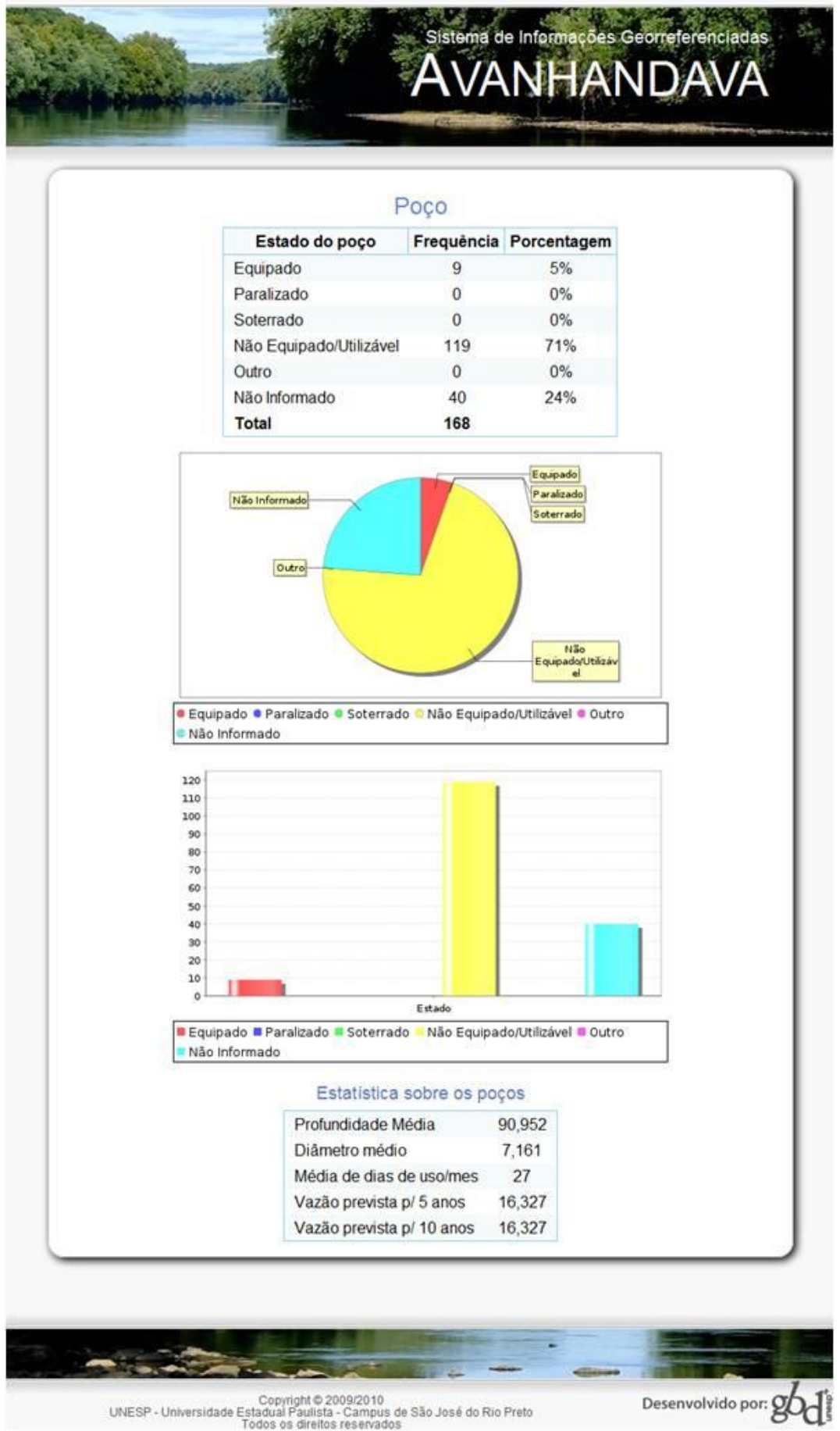

Figura 4. Exemplo de relatório convencional de poço 
No trabalho de campo, também executado como parte dos objetivos do projeto, observou-se que toda propriedade tem, pelo menos, um poço semi-artesiano, sem proteção e que o manejo correto para a preparação e aplicação dos defensivos agrícolas utilizados na cultura da laranja é uma deficiência constatada in loco. A falta de equipamentos de proteção individual, manejo correto na mistura dos produtos químicos e destinação correta das embalagens podem comprometer a estrutura hídrica da sub-bacia do Avanhandava. A maneira com se formam os pomares, não obedecendo às técnicas adequadas para preservar a área da erosão, também é um problema inicial.

Um caso presenciado é de uma propriedade onde é realizada coleta de material reciclável, na qual o poço da propriedade está "misturado" com as "sobras" do material que não é comercializado e o entulho compromete a margem e a nascente.

Durante o trabalho de campo, também foi constatado que as nascentes não estão protegidas. O Rio Avanhandava está assoreado e as estradas antigas servem de "esgoto" para canalizar a enxurrada em direção aos rios mais próximos quando chove, mostrado na figura 5. Nas figuras 5(a) e 5(b), estão evidentes que o assoreamento é um caso constatado nas estradas da região, que não fica restrito apenas às estradas, mas também ocorrem às margens do rio, como visto na figura 5 (c).

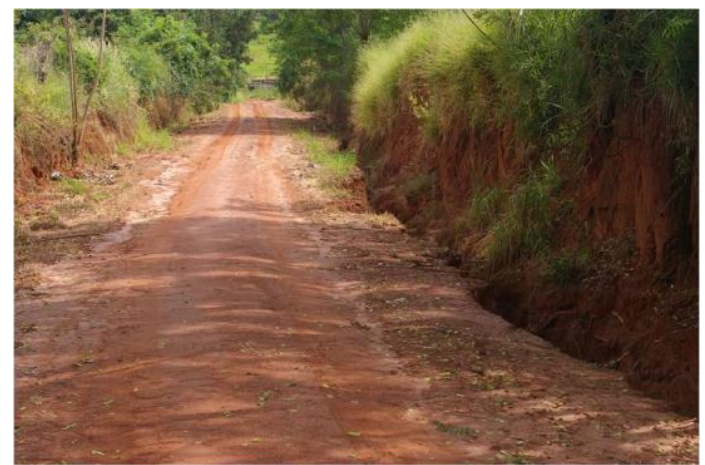

(a)

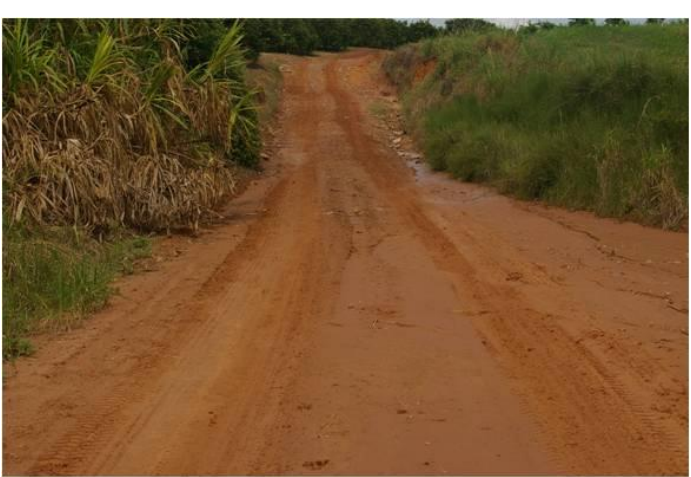

(b) 


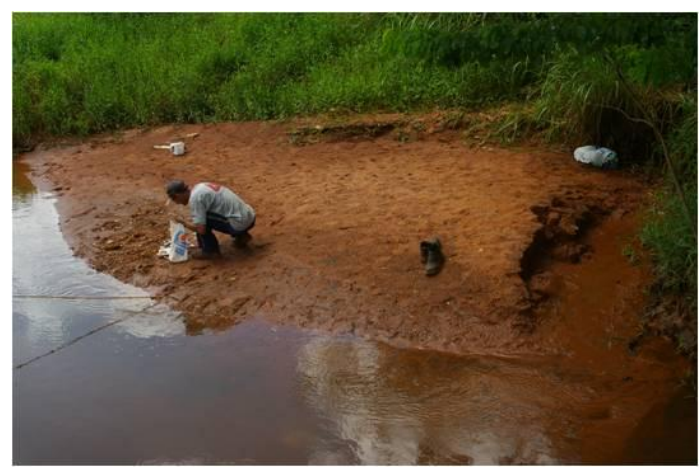

(c)

Figure 5. Assoreamento nas estradas, à margem do rio e trecho do rio agonizando

Além disso, os pomares estão sendo plantados sem obedecer ao nível do terreno, como é visto na figura 6 . Até atingir a idade adulta, as ruas canalizam a enxurrada em direção aos carreadores, enquanto não formam uma proteção vegetal minimizando o problema. Além dos problemas naturais que estão ocorrendo, algum rio próximo está servindo de depósito de lixo de construção civil.

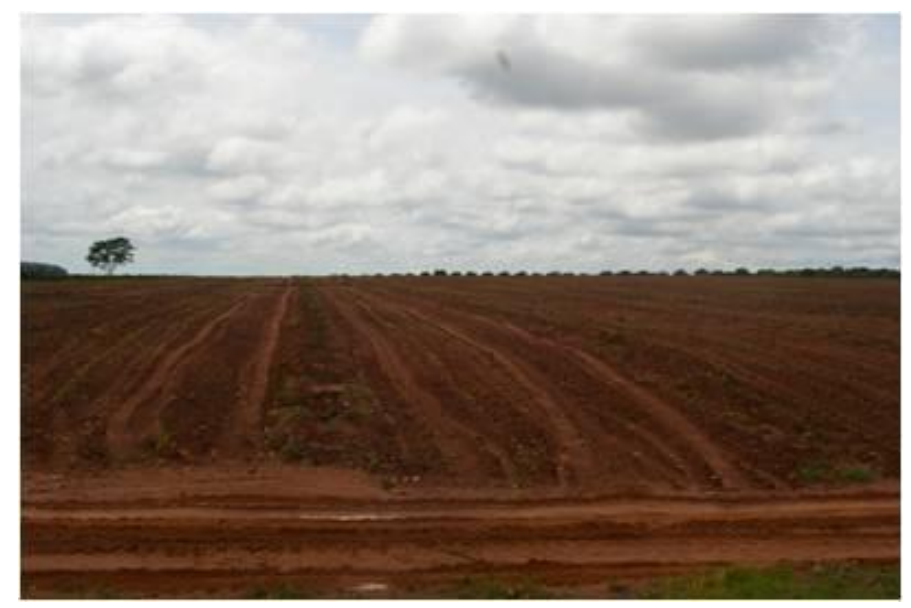

Figura 6. Cultivo com ausência de curvas de níveis

Diante das informações extraídas do sistema computacional e análise dos dados coletados, conclui-se que dos chefes de família das propriedades visitadas, 65,48\% tem apenas o primário completo e $16,97 \%$ utilizam o recurso hídrico para irrigação. Com relação aos poços, apenas 5,36\% são equipados. Para o controle da erosão, 20,36\% utilizam curvas de nível e 55,09\%, faixa vegetal. 
Dentre os resultados, o que é mais alarmante é a situação administrativa da captação d' água. 92,26\% não possuem autorização.

Além dos resultados e conclusões descritos, observou-se que o cultivo da cana-deaçúcar está, aos poucos, mudando o panorama da sub-bacia, obedecendo todas as técnicas de preparo do solo para o plantio. No setor da citricultura, o preço da caixa da laranja comercializada nos últimos anos não motiva mais os citricultores e os grandes investimentos em irrigação estão abandonados, devido à energia elétrica que eleva o custo na produção da laranja.

\section{CONCLUSÕES}

O trabalho desenvolvido oferece grande contribuição no suporte e apoio aos órgãos responsáveis pela gestão dos recursos hídricos na tomada de decisão. O casamento entre o sistema computacional e o trabalho de campo executado é o principal diferencial e fator importante para a obtenção dos resultados.

A coleta de dados in loco possibilitou, ainda, uma caracterização da região abordada mais ampla, como exemplo, casos de irregularidades que prejudicam não só o recurso hídrico, mas também a saúde do trabalhador durante a pulverização de defensivos agrícolas, assim como o caso de assoreamento das estradas e ausência de curvas de nível nos cultivos.

Por meio das informações apresentadas pelo sistema e os resultados do trabalho de campo, em um sentido imediatista e em longo prazo, faz-se necessária uma campanha de formação e esclarecimento a todos os profissionais envolvidos na citricultura e a intervenção dos órgãos públicos nas estradas, que se encontram passivas de enxurradas que carregam resíduos e, consequentemente, assoreando o rio Avanhandava. 


\section{REFERÊNCIAS}

CBH-TG103. Deliberação CBH-TG № 103/2004 de 15/12/2004. Disponível em <http:// www.sigrh.sp.gov.br/sigrh/ARQS/DELIBERACAO/CRH/CBH-TG/1761/delcbhtg103-4\%20\%20bacia\%20critica.doc>. Acesso em: 20 jul. 2010.

CBH-TG141. Deliberação CBH-TG № 141 de 25/03/2008. Disponível em <http://www.sigrh.sp.gov.br/sigrh/ARQS/DELIBERACAO/CRH/CBH-TG/2558/delcbhtg14108_\%20prioridade\%20de\%20investimentos\%20fehidro-2008.pdf>. Acesso em: 20 jul. 2010.

DEPPE, Flavio., PAULA, Eduardo Vedor, PRATES, José Eduardo. Monitoramento de incêndios e do estado da vegetação através da utilização de imagens NOAA/AVHRR, Anais do XI SBSR, Belo Horizonte, abril de 2003, INPE, 453-460.

FRANCO, Renato Alberto Momesso. Qualidade da Água para Irrigação na Microbacia do Córrego do Coqueiro no Noroeste Paulista. Ilha Solteira: Universidade Estadual Paulista "Júlio de Mesquita Filho", 2008. Dissertação de mestrado, Agronomia, Ilha Solteira, 2008.

GÜTING, Ralf Hartmut. An introduction to spatial database systems. The VLDB Journal, v. 3, n. 4, p. 357-399, 1994.

KEISLER, Jeffrey; BLAKE, Roger; WAGNER, Janet. Implementing Geographic Information Systems (GIS) in Spreadsheet Models: What, Why, and How, hicss, 42nd Hawaii International Conference on System Sciences, pp.1-10, 2009.

LARMAN, C.: Applying UML and Patterns: An Introduction to Object-Oriented Analysis and Design and Iterative Development. Bookman, 2nd ed.

MACLACHLAN, John. C. et al. Mapping health on the Internet: A new tool for environmental justice and public health research. Health \& Place, v. 13, n. 1, p. 72-86, March 2007. ISSN 1353-8292.

NOON, Charles E.; HANKINS, Charles T. Spatial data visualization in healthcare: supporting a facility location decision via GIS-based market analysis, Proceedings of the 34th Annual Hawaii International Conference on System Sciences, pp. 1 - 10, Jan. 2001. 
OLIVEIRA, Ivani Matos, ZEILHOFER, Peter, SANTOS, Emerson Soares. Segmentação para classificação de áreas urbanas a partir de imagem digital do Landsat7/ETM+: Estudo de caso - Cuiabá-MT, XIII SBSR, Florianópolis, abril de 2007, INPE, p. 60116018.

RAO, Mahesh et al. A web-based GIS Decision Support System for managing and planning USDA's Conservation Reserve Program (CRP). Environmental Modelling \& Software, v. 22, n. 9, p. 1270-1280, setembro 2007. ISSN ISSN 1364-8152.

RISPA Rede Integrada de Informações para a Saúde. Conceitos Básicos de Sistemas de Informação Geográfica e Cartografia aplicados à saúde. Brasília: Organização Panamericana da Saúde/Ministério da Saúde, 2000.

SILVA, C. R., SILVA, M. R., RIBEIRO, M. Análise temporal da variação da mata ciliar do rio São Francisco na região do norte de Minas Gerais com base nas imagens do sensor CCD do CBERS, COBRAC, Florianópolis, outubro de 2006. 